\title{
Celiac Disease and the Gluten-free Diet: Registered Dietitian Nutritionists' Self-reported Knowledge Varies
}

\author{
Joan Geiger ${ }^{1}$, Yeong Rhee ${ }^{1}$, Sherri N. Stastny ${ }^{1, *}$, Ardith Brunt ${ }^{1}$, Elizabeth Blodgett Salafia ${ }^{2}$ \\ ${ }^{1}$ Health, Nutrition, and Exercise Sciences, North Dakota State University, Fargo, United States of America \\ ${ }^{2}$ Human Development and Family Science, North Dakota State University, Fargo, United States of America \\ *Corresponding author: Sherri.stastny@ndsu.edu
}

\begin{abstract}
Background: Adherence to the gluten-free diet is the only treatment for celiac disease (CD) and some patients report lack of expertise among registered dietitian nutritionists (RDNs). Objective: To measure RDN self-reported celiac disease (CD) knowledge and preferences for resources for self-education and patient education. Methods: A cross-sectional research design was used to recruit RDNs from participating state affiliates Alaska, Colorado, Connecticut, Delaware, Montana, Nebraska, and North Dakota in 2013. A 35 item internet-based survey was distributed via Survey Monkey and included Likert scale questions based on a previous RDN allergy survey. Demographic and gluten-free diet and CD multiple-choice questions were also included. Results: Four-hundred and five registered dietitians (RDNs) who volunteered from the seven participating affiliates responded to the survey. Chi-squares were used to determine frequencies and differences in responses per variable. Pearson correlation analyses were performed to determine the correlation of age, education level, years of practice, or number of CD patients seen per week and CD self-reported knowledge. RDNs reported either moderate or high levels of knowledge for all seven self-reported knowledge topics. Over $85 \%$ of RDNs selected correct answers for five CD knowledge questions. Professional and academic publications were the most commonly used resources by RDNs for self-education whereas handouts were the most commonly used resource by RDNs for patient education. Conclusion: RDNs may need more reinforcement on identification and treatment of nutritional deficiencies of CD management.
\end{abstract}

Keywords: Celiac Disease, Registered Dietitian Nutritionist, Celiac Disease Knowledge, gluten free diet, gluten free diet knowledge

Cite This Article: Joan Geiger, Yeong Rhee, Sherri N. Stastny, Ardith Brunt, and Elizabeth Blodgett Salafia, "Celiac Disease and the Gluten-free Diet: Registered Dietitian Nutritionists' Self-reported Knowledge Varies." International Journal of Celiac Disease, vol. 5, no. 2 (2017): 56-62. doi: 10.12691/ijcd-5-2-1.

\section{Introduction}

Approximately one to two percent of individuals worldwide are affected by celiac disease (CD) with an increased prevalence among whites [1,2]. Adherence to the gluten-free diet (GFD) is the only treatment for CD as ingestion of even trace amounts of gluten can induce small-bowel damage [3]. The GFD can be quite restrictive and therefore difficult to follow. This is partially due to increased cost and limited availability of gluten-free products, taste/texture of products, the complexity of the diet, and its restrictive nature [4]. Therefore, a visit with a skilled Registered Dietitian Nutritionist (RDN) is considered an essential aspect of managing the disease $[2,5,6]$.

Information regarding $\mathrm{CD}$ can be obtained from numerous technology sources including Internet web sites, smart phone applications, and social media. Despite the abundance of information, not all of the information is accurate. An evaluation of $98 \mathrm{CD}$ websites revealed that $47(48 \%)$ of the websites provided information that was less than $95 \%$ accurate [7]. The Academy of Nutrition and Dietetics (AND) reviewed 10 free iPhone apps for gluten-free eating on a scale of one to five stars (five stars being the best). Of these apps one received one star, five received two stars, two received three stars, one received four stars, and one received four and one half stars [8]. Since following a GFD is the only treatment for CD disease, education and a visit with a skilled RDN are identified as key essentials in CD management. It is imperative that RDNs are able to provide the patient with accurate and up-to-date information.

There is limited recent research regarding how skilled RDNs are with CD management. For example, in a survey of CD patients who worked with an RDN, about $54 \%$ of participants reported that RDNs did not seem to be knowledgeable about $\mathrm{CD}$ and $53 \%$ did not find RDNs to be helpful [9]. The RDN is the nutrition expert and although management of patients with CD may be an area of specialty, every RDN should minimally know where to turn for reliable science-based information when needed. For example, CD customers in RDN-led restaurants (especially, for example, in a healthcare setting) rely upon careful food handling (i.e., no kitchen cross contamination with gluten containing and GF foods). RDNs who work in the field of education must understand the importance of 
careful consideration of allergy-education in various coursework.

As mentioned, there is evidence that some of the CD information available to patients is not accurate, and little is known regarding overall RDN knowledge of CD management. The purpose of this study was to measure RDN self-rated and other CD knowledge and preference for resources for $\mathrm{CD}$.

\section{Materials and Methods}

\subsection{Subjects}

The survey was sent electronically to all members of each AND state affiliate that agreed to participate in the research: Alaska, Colorado, Connecticut, Delaware, Montana, Nebraska, and North Dakota in 2013. Researchers contacted statewide AND groups to request their assistance in distributing recruitment emails to RDNs. A researcher-scripted message was sent by respective state affiliates inviting RDNs to complete the survey. If they chose to participate, they clicked on the link that brought them to Survey Monkey (Palo Alto, CA). Participants were informed that choosing to complete the survey implied consent and agreement to participate in the study. Affiliates were asked to resend the survey a second time in 2013 to increase response.

Before the final survey was released, a small group of upper level dietetic students $(n=16)$ was asked to answer the survey questions and to give feedback regarding ease of use, ease and time to completion, and suggestions for improved understandability and readability in order to develop the final survey used for the study. The testing did reveal the need to re-word several questions. After the survey was finalized, approval from the University Institutional Review Board was obtained before this survey administration.

\subsection{Survey Design}

The survey used for this cross-sectional study was created to identify RDN self-rated and other CD knowledge and preference for resources for CD. The survey included 35 possible items. The tool was modeled after a survey used to measure self-reported proficiency of RDNs and food allergy management [10]. Survey topics included demographics, seven self-rated CD knowledge topics using a 4-point Likert scale ("high", "moderate", "low", and "not at all"), five multiple choice CD knowledge questions and finally probed for $C D$ management resources used for self-education and for patient education.

\subsection{Statistical Analysis}

The collected data were analyzed using SAS (version 9.3; SAS Institute Inc., Cary, NC). Chi-squares were determined to estimate frequencies and differences in responses per variable. Categories were aggregated (e.g. practice setting) for selected variables to avoid problems with underlying assumptions of the chi-square tests of independence. Pearson correlation analyses were performed to determine the correlation of the independent variables, age, education level, years of practice, or number of CD patients seen per week, compared to the dependent variables, RDN CD skill self-rating and other CD knowledge. The significance level used for each statistical analysis was $P<0.05$.

\section{Results}

Of the 405 RDNs who participated in the study, 98\% $(\mathrm{n}=378)$ identified themselves as female. According to CDR, $1.6 \%$ of RDNs were male, so this is comparable to the participants who completed the current survey [11]. In 2013, there were 5,672 RDNs registered with CDR [11]. It is unknown how many members were part of the participating states, so the potential response rate is also unknown. As participants were not required to answer all questions of the survey, some of the questions had fewer than 405 responses. Other characteristics including area of practice are displayed in (Table 1)

Table 1. Demographics of a sample of 405 registered dietitian nutritionists who completed a celiac disease survey

\begin{tabular}{lcc}
\hline & $\%$ & $\mathrm{n}$ \\
\hline Sex $(\mathrm{n}=386)^{\mathrm{a}}$ & 2 & \\
Male & 98 & 378 \\
Female & &
\end{tabular}

$\begin{array}{lcc}\text { Age }(\mathrm{n}=366)^{\mathrm{a}} & & \\ \text { 20-29 years } & 17 & 61 \\ 30-39 \text { years } & 26 & 94 \\ 40-49 \text { years } & 15 & 55 \\ 50-59 \text { years } & 28 & 104 \\ 60-69 \text { years } & 13 & 47 \\ 70+\text { years } & 1 & 5 \\ & & \\ \text { Education level }(\mathrm{n}=395)^{\mathrm{a}} & & \\ \text { Bachelor's degree } & 53 & 209 \\ \text { Master's degree } & 43 & 169 \\ \text { Doctorate degree } & 4 & 17 \\ & & \\ \text { Years of practice }(\mathrm{n}=398)^{\mathrm{a}} & & 122 \\ 0-5 \text { years } & 30 & 63 \\ 6-10 \text { years } & 16 & 38 \\ 11-15 \text { years } & 10 & \\ 15+\text { years } & 44 & \end{array}$

Number of CD patients seen per week ${ }^{\mathrm{a}}$

$\begin{array}{lcc}0 & 63 & 251 \\ 1-3 & 34 & 133 \\ 4-6 & 3 & 10 \\ 7-9 & <1 & 1 \\ 10+ & <1 & 2\end{array}$

\begin{tabular}{lcc} 
Practice setting $^{\mathrm{a}}$ & & \\
Outpatient & 31 & 143 \\
Inpatient & 25 & 117 \\
Private Practice & 6 & 30 \\
Food Service & 11 & 47 \\
Other & 28 & 130 \\
\hline
\end{tabular}

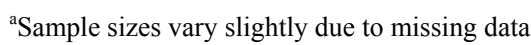


In terms of practice setting, private practice was identified having the fewest participant RDNs with $6 \%$ $(n=30)$ compared to outpatient or other, which $31 \%$ $(n=143)$ and $28 \%(n=130)$ of RDNs reported, respectively (Table 1). Long-term care, government research center, academics, rural health, public health, WIC, retail grocery, or extension were listed as other settings of practice.

RDNs' self-reported knowledge in several areas of CD management was collected. RDNs primarily rated themselves "high" for gluten-free definitions (65\%), determining if a food contains gluten by reading nutrition labels (61\%), understanding CD (55\%), and providing avoidance education (51\%). RDNs primarily rated themselves "moderate" for being able to provide information regarding local glutenfree support groups $(31 \%)$, identifying potential nutrition risks associated with a gluten-free diet, and providing information regarding local gluten-free restaurants or grocery stores (45\%). A significant negative correlation of
RDNs' self-reported knowledge and number of CD patients seen per week was found for each of the seven questions. A significant negative correlation of RDNs' self-reported knowledge and years of practice was found for two of the seven questions (able to provide avoidance education, $\mathrm{r}=-0.321, P<0.0001$; understands $\mathrm{CD}$ definition, $\mathrm{r}=-0.146, P=0.004)$. A significant negative correlation of RDNs' self-reported knowledge and age was found for three of the seven questions (understands CD definition, $\mathrm{r}=-0.138, P=0.007$; able to provide avoidance education, $\mathrm{r}=-0.317, \quad P<0.0001$; able to provide information regarding local gluten-free establishments, $\mathrm{r}=-0.100$, $P<0.05)$. No significant correlation was found with RDNs self-reported knowledge and education level (Table 2).

A series of five multiple choice $\mathrm{CD}$ questions were coded as either correct or incorrect and compared to age, education, practice years, and CD patients seen per week (Table 3).

Table 2. Registered dietitian nutritionist survey participants' self-reported knowledge celiac disease as either high, moderate, low or none at all

\begin{tabular}{|c|c|c|c|c|}
\hline \multirow[t]{2}{*}{ Proficiency Area } & \multicolumn{4}{|c|}{ Self-Reported Proficiency Level } \\
\hline & High \% (n) & Moderate\% (n) & Low \% (n) & None at all \% (n) \\
\hline Able to determine if food item contains gluten using nutrition label & $61(233)$ & $36(139)$ & $3(13)$ & $0(0)$ \\
\hline Understands celiac disease definition & $55(213)$ & $41(157)$ & $4(16)$ & $0(0)$ \\
\hline $\begin{array}{l}\text { Able to provide avoidance education } \\
\text { (e.g., cross-contamination, ingredient identification) }\end{array}$ & $51(197)$ & $40(158)$ & $8(29)$ & $1(3)$ \\
\hline $\begin{array}{l}\text { Able to provide information regarding local restaurants or grocery } \\
\text { stores that provide gluten-free items }\end{array}$ & $33(128)$ & $45(178)$ & $20(76)$ & $2(8)$ \\
\hline $\begin{array}{l}\text { Able to identify nutrients at risk for deficiency when following a } \\
\text { gluten-free diet }\end{array}$ & $32(125)$ & $50(194)$ & $17(29)$ & $1(3)$ \\
\hline $\begin{array}{l}\text { Able to provide information regarding local gluten-free diet support } \\
\text { groups }\end{array}$ & $30(117)$ & $31(121)$ & $26(100)$ & $13(47)$ \\
\hline
\end{tabular}

Table 2 (continued). Correlation of registered dietitian nutritionists self-reported knowledge of celiac disease (CD), on a 4-point Likert scale, compared to age, level of education, years of practice and number of CD patients seen per week ${ }^{1}$

\begin{tabular}{|c|c|c|c|c|}
\hline & Age & Education & Practice Years & CD Pts./Week2 \\
\hline Understands gluten-free definition & $\begin{array}{l}0.089 \\
0.082 \\
3852\end{array}$ & $\begin{array}{c}0.0301 \\
0.558 \\
381\end{array}$ & $\begin{array}{c}-0.089 \\
0.082 \\
384\end{array}$ & $\begin{array}{c}-0.189 \\
0.0002 \\
384\end{array}$ \\
\hline Able to determine if food item contains gluten using nutrition label & $\begin{array}{c}0.028 \\
0.585 \\
384\end{array}$ & $\begin{array}{c}-0.039 \\
0.450 \\
380\end{array}$ & $\begin{array}{c}0.040 \\
0.441 \\
383\end{array}$ & $\begin{array}{c}-0.155 \\
0.002 \\
382\end{array}$ \\
\hline Understands celiac disease definition & $\begin{array}{c}-0.138 \\
0.007 \\
387\end{array}$ & $\begin{array}{c}-0.048 \\
0.344 \\
383\end{array}$ & $\begin{array}{c}-0.146 \\
0.004 \\
386\end{array}$ & $\begin{array}{c}-0.238 \\
<.0001 \\
385\end{array}$ \\
\hline Able to provide avoidance education & $\begin{array}{c}-0.317 \\
<.0001 \\
385\end{array}$ & $\begin{array}{c}0.018 \\
0.726 \\
381\end{array}$ & $\begin{array}{c}-0.321 \\
<.0001 \\
384\end{array}$ & $\begin{array}{c}-0.266 \\
<.0001 \\
383\end{array}$ \\
\hline $\begin{array}{l}\text { Able to provide information regarding local restaurants or grocery } \\
\text { stores } 3\end{array}$ & $\begin{array}{c}-0.100 \\
0.0499 \\
384\end{array}$ & $\begin{array}{c}0.004 \\
0.937 \\
380\end{array}$ & $\begin{array}{c}-0.066 \\
0.199 \\
383\end{array}$ & $\begin{array}{c}-0.217 \\
<.0001 \\
382\end{array}$ \\
\hline Able to identify nutrients at risk for deficiency & $\begin{array}{c}-0.024 \\
0.639 \\
385\end{array}$ & $\begin{array}{c}-0.040 \\
0.436 \\
381\end{array}$ & $\begin{array}{c}-0.041 \\
0.424 \\
384\end{array}$ & $\begin{array}{c}-0.157 \\
0.002 \\
383\end{array}$ \\
\hline $\begin{array}{l}\text { Able to provide information regarding local gluten-free diet support } \\
\text { groups }\end{array}$ & $\begin{array}{c}-0.089 \\
0.082 \\
386\end{array}$ & $\begin{array}{c}-0.037 \\
0.477 \\
382\end{array}$ & $\begin{array}{c}-0.098 \\
0.054 \\
385\end{array}$ & $\begin{array}{c}-0.193 \\
0.0001 \\
384\end{array}$ \\
\hline
\end{tabular}

${ }^{1}$ Each cell indicates $\mathrm{r}$ ( $\mathrm{r}=$ Pearson Correlation Coefficient), p-value, and $\mathrm{n} ;{ }^{2}$ Number of celiac disease patients seen per week; ${ }^{3}$ Able to provide information regarding local restaurants or grocery stores that provide gluten-free it. 
Table 3. Registered dietitian nutritionists' age, education, years of practice, or number of CD patient seen per week compared to correct or incorrect answers for five multiple-choice CD knowledge questions 1

\begin{tabular}{|c|c|c|c|c|}
\hline & Age & Education & Practice Years & CD Pts./Week ${ }^{2}$ \\
\hline Which of the following indicates the presence of gluten? & $\begin{array}{c}-0.037 \\
0.472 \\
377\end{array}$ & $\begin{array}{c}-0.078 \\
0.131 \\
373\end{array}$ & $\begin{array}{c}-0.014 \\
0.781 \\
376\end{array}$ & $\begin{array}{c}0.082 \\
0.114 \\
375\end{array}$ \\
\hline Which of the following would be considered gluten-free? & $\begin{array}{c}-0.058 \\
0.262 \\
377\end{array}$ & $\begin{array}{c}-0.007 \\
0.898 \\
373\end{array}$ & $\begin{array}{c}-0.045 \\
0.390 \\
376\end{array}$ & $\begin{array}{c}0.019 \\
0.712 \\
375\end{array}$ \\
\hline A gluten-free diet may be low in the following nutrients & $\begin{array}{c}0.063 \\
0.227 \\
376\end{array}$ & $\begin{array}{c}0.011 \\
0.840 \\
372\end{array}$ & $\begin{array}{c}0.020 \\
0.698 \\
375\end{array}$ & $\begin{array}{c}0.046 \\
0.373 \\
374\end{array}$ \\
\hline Supplements may contain fillers from wheat or barley & $\begin{array}{c}-0.028 \\
0.591 \\
378\end{array}$ & $\begin{array}{c}-0.040 \\
0.440 \\
374\end{array}$ & $\begin{array}{c}-0.030 \\
0.557 \\
377\end{array}$ & $\begin{array}{c}-0.018 \\
0.728 \\
376\end{array}$ \\
\hline In order to be diagnosed, gluten-free diet must be maintained for two months & $\begin{array}{c}0.145 \\
0.0047 \\
378\end{array}$ & $\begin{array}{c}0.0003 \\
0.995 \\
374\end{array}$ & $\begin{array}{c}0.174 \\
0.0007 \\
377\end{array}$ & $\begin{array}{c}0.005 \\
0.915 \\
376\end{array}$ \\
\hline
\end{tabular}

${ }^{1}$ Each cell indicates $\mathrm{r}\left(\mathrm{r}=\right.$ Pearson correlation coefficient), $\mathrm{p}$-value, and $\mathrm{n} ;{ }^{2}$ Indicates CD patients seen per week.

For the first multiple-choice question, "Which of the following indicate the presence of gluten?" the correct answer was selected by $94 \%$ of participants $(n=354)$. For the second question, "Which of the following ingredient lists would be considered gluten-free?" the correct answer was selected by $94 \%$ of respondents $(n=355)$. For the third question, "A gluten-free diet may be low in the following nutrients: iron, calcium, fiber, B-vitamins," the correct answer was selected by $85 \%$ of participants $(n=321)$. For the fourth question, "Supplements and pharmaceuticals may contain fillers made from wheat or barley," the correct answer was selected by $98 \%$ of participants $(n=372)$. For the fifth question, "In order to be diagnosed with $\mathrm{CD}$, the patient must first be following a GFD for at least two months," the correct answer was selected by $90 \%$ of participants $(n=339)$. There was a significant difference in RDNs' knowledge and number of CD patients seen per week for one of the five questions (indicating which item contained gluten, $P=0.015$, data not shown). There was a significant difference in RDNs' knowledge and years of practice for one of the five questions (length of time needed to follow gluten-free diet before $\mathrm{CD}$ diagnosis, $P<0.005$, data not shown). There was a significant difference in RDNs' knowledge and age in the aforementioned question (length of time needed to follow gluten-free diet before CD diagnosis, $P=0.004$, data not shown). There was no significant difference in RDNs' knowledge and education level. There was a significant correlation for RDNs' knowledge in only one question (length of time needed to follow gluten-free diet before $C D$ diagnosis can be accepted) with their age $(P<0.005)$ and years of practice $(P<0.001)$. No significant correlations were found for RDNs' knowledge and education level or number of CD patients seen per week for any of the questions.

RDNs were asked to select all resources that they have used in the past to receive information about $\mathrm{CD}$ in addition to selecting all resources that they have used to educate patients. Academic publications were the most frequently used resource to educate self $(66 \%, n=266)$ while handouts were the most frequently used resource to educate patients $(78 \%, \mathrm{n}=316)$.

\section{Discussion}

Overall, this study was designed to explore relationships among RDN self-rated and other CD knowledge and preference for resources for CD education. We found that RDNs rated themselves as quite knowledgeable about CD and the GFD but not knowing how to find supports for patients. The results included moderate or high knowledge for all seven of the seven topics with the highest percentage $(65 \%)$ of RDNs reporting a high level for knowing the $\mathrm{CD}$ definition and the lowest percentage $(30 \%)$ of RDNs reporting a high level of understanding of local CD support groups. Previous research is somewhat limited regarding RDNs' self-reported knowledge of CD, but in a survey of RDNs who work with children with food allergies, most respondents $(>50 \%)$ rated themselves less than high proficiency in all areas aside from understanding the definition of food allergies and food intolerance and very few respondents $(<20 \%)$ rated their level high for understanding the steps involved in a food allergy diagnosis, developing an elimination diet, and evaluating safe food items in a hospital or school setting. [10]

Results varied by RDN level of experience, as indicated by number of CD patients seen per week. We found a significant negative correlation of RDNs' self-reported knowledge and number of CD patients seen per week for all self-reporting knowledge questions translating to a decrease in self-reported knowledge with an increase in the number of CD patients seen per week. This finding could be related to the sample's CD exposure, as $63 \%$ reported seeing no $\mathrm{CD}$ patient per week on average, while $39 \%$ of sample saw greater than one CD patient per week. Additionally, a significant negative correlation of RDNs' self-reported knowledge and years of practice was found for two of the questions; this again could be attributed to unbalanced distribution of groups for CD patients seen per week was somewhat unbalanced.

Since meeting with a skilled RDN has been identified as an essential aspect of managing CD [5], study methods were designed to estimate RDNs' knowledge of CD by asking five multiple-choice questions. In a study of 
biopsy-diagnosed CD patients, $40 \%$ of patients $(n=164)$ agreed with the statement that it is difficult to find an RD knowledgeable about the GFD [12]. In a similar study which surveyed 160 biopsy-diagnosed CD patients, 54\% of the participants $(n=86)$ reported that RDNs did not seem to be knowledgeable about $\mathrm{CD}$, and $53 \%$ of patients $(\mathrm{n}=85)$ did not find RDNs to be helpful [9].

While our results indicate that RDNs have a fairly good understanding of $\mathrm{CD}$ topics, it is imperative that RDNs and the medical team are able to provide the $\mathrm{CD}$ patient with as much reliable information as possible. Identification and treatment of nutritional deficiencies has been identified as one of the essential aspects of managing $\mathrm{CD}$ [5], and we found that RDNs may need more reinforcement of this topic based on self-reported knowledge levels and knowledge question findings.

Handouts and academic publications were the conventional resources that RDNs used most frequently to educate patients with $\mathrm{CD}$. In a similar study where RDNs were surveyed regarding food allergies, handouts were also identified as the leading resource used to educate patients [10]. In terms of patient preference for methods of receiving CD education, a German study of 64 biopsy-diagnosed $\mathrm{CD}$ patients found that the use of a computer-based interactive training program significantly increased knowledge and sustainability of CD topics as compared to a conventional training for CD patients [13], so future studies should explore the effectiveness of using such programs for CD education.

The internet was the preferred technology resource for educating a patient with $\mathrm{CD}$ as well as the preferred technological resource for self-education. While the current study did not determine specific website preferences of RDNs, there are several resources available to RDNs and general public to increase CD knowledge. For example, the Academy of Nutrition and Dietetics' website hosts information for academy members including its Evidence-Based Celiac Disease Practice Guidelines and Evidence Analysis Library. Additionally, the website provides information to the public on various $\mathrm{CD}$ topics such as gluten-free app reviews $[8,14]$.

There is evidence that there are some CD websites that lack validity and reliability of information and others that are valid, reliable and up-to-date. According to a study in which researchers determined the accuracy, comprehensiveness, transparency, and readability of various CD websites, only 4 of 98 (4\%) websites were found to be trustworthy and reliable; of those, two were considered nonprofit websites (http://www.celiac.org and http://www.digestive.niddk.nih.gov/ddiseases/pubs/celiac), one academic (http://celiaccenter.uscd.edu), and one commercial (http://celiacdisease.about.com) [7]. Other websites such as the CD Foundation ${ }^{15}$ or National Institute of Health [16] also provide information on CD topics which can improve the general public or RDNs knowledge base of CD topics.

It has been estimated that $55 \%$ of those over the age of 18 look to the Internet as a source of health or medical information [17], and research has supported the notion of utilizing Internet-based programs for health interventions. The use of eHealth, which can be defined as using technology with an emphasis on the Internet to communicate and monitor health-related practices, [18] has been found to be effective with delivering health interventions. For example, in a study in which 36 volunteers participated in a 12 -week intervention, which consisted of 36 healthy lifestyle-focused email messages, participants reported increased intake of fiber, decreased intake of fat, and increased physical activity levels. Additionally, overweight and obese participants lost an average of eight pounds during the intervention period [19].

A strength of this study is the inclusion of RDN's from the entire CDR membership of seven geographically different states. That RDNs were included from all areas of practice was intentional and could be noted as a strength of the study-all RDNs need to either have knowledge or know where to get reliable information about $\mathrm{CD}$ because the RDN is considered the nutrition expert and $\mathrm{CD}$ is a very common issue.

There were several limitations with this research. First, the overall response rate was low despite repeated reminders to potential participants. The length of the survey and limited availability may have prevented some participation. Second, there was a lack of sex diversity among RDN participants but both were similar to overall RDN membership. Third, while CD knowledge and awareness is important among RDNs, some RDNs with limited exposure to $\mathrm{CD}$ may have struggled on the survey knowledge questions. Sixty-three percent of the RDN sample reported seeing zero CD patients a week, so the majority of participants seldom work with a patient with $\mathrm{CD}$, thus limiting determination about RDNs who work with the CD patient regularly. Additionally, if RDNs worked in one of the settings in which CD exposure is low, she or he might not have been interested in fully completing the survey.

In conclusion, for this study, most RDNs self-rated GFD and CD knowledge as high and those RDNs who reported seeing more patients per week had the highest levels. Only a moderate number of RDNs reported knowing where those following a GFD could shop or eat out. Most RDNs in this study reported using academic publications above all sources to increase knowledge of the GFD and CD. As academic publications, the Internet, and self-taught methods were the resources most frequently used by RDNs to learn about $\mathrm{CD}$, future research might be beneficial to determine the accuracy of such resources. Additionally, future studies regarding celiac disease patients learning preferences would be of interest.

Future studies might also be beneficial in determining the best way to provide CD education to RDNs. Research has suggested that more medical programs are encouraging the use of apps, but the accuracy of some apps can range in accuracy from $48-55 \%$, so it is important for RDNs and medical team to be able to determine whether or not an app is reliable [20].

Topics of which the $\mathrm{CD}$ patient needs to be informed are the nutritional quality of the GFD and preventing contamination with gluten-containing foods [21]. Patients should also be trained to monitor for signs and symptoms of $\mathrm{CD}$ related problems and how individual responses vary. The five knowledge based questions of the survey revealed some potential knowledge gaps, as no question resulted in $100 \%$ correct responses from the participating 
RDNs. While a goal of $100 \%$ accuracy is quite high, the research shows that even the smallest ingestion of gluten can induce intestinal damage [3], so it is imperative the patient receive accurate information from RDNs. RDNs may need more reinforcement on identification and treatment of nutritional deficiencies of CD management.

\section{Acknowledgements}

The authors thank Curt Doetkott and Kayeromi Gomez for aiding in statistical analyses.

\section{References}

[1] Autodore JR, Verma RM, Gupta KB. Celiac disease and its treatment. Top Clin Nutr. 2012;27(3):270-276.

[2] Niewinski M. Advances in celiac disease and gluten-free diet. $J$ Am Diet Assoc. 2008;108(4):661-672.

[3] Lähdeaho M, Mäki M, Laurila K, Huhtala H, Kaukinen K. Smallbowel mucosal changes and antibody responses after low- and moderate-dose gluten challenge in celiac disease. $B M C$ Gastroenterol. 2011;11:129.

[4] Hall N, Rubin G, Charnock A. Systematic review: adherence to a gluten-free diet in adult patients with coeliac disease. Aliment Pharmacol Ther. 2009;30(4):315-330.

[5] National Institute of Health (2004). Consensus Development Conference Statement. Paper presented at the NIH Consensus Development Conference on Celiac Disease. 2004. http://consensus.nih.gov/2004/2004CeliacDisease118html.html. Accessed May 31, 2016.

[6] Case S. The gluten-free diet: how to provide effective education and resources. Gastroenterology. 2005;128 (4 Suppl. 1):128-134.

[7] McNally S, Donohue M, Newton K, Ogletree S, Conner K, Ingegneri S, Kagnoff $M$. Can consumers trust web-based information about celiac disease? Accuracy, comprehensiveness, transparency, and readability of information on the internet. Interact J Med Res. 2012;1(1).
[8] Crandall, J. (2012). Gluten-free app reviews. http://www.eatrightpro.org/resources/media/trends-andreviews/app-reviews. Accessed August 2, 2016.

[9] Tidwell D, Bomba A. Attitudes of people with celiac disease towards dietitians and medical nutrition therapy. J Am Diet Assoc. 2001;101(95):29.

[10] Groetch M, Christie L, Vargas P, Jones S, Sicherer S. Food allergy educational needs of pediatric dietitians: a survey by the Consortium of Food Allergy Research. J Nutr Educ Behav. 2010;42(4):259-264.

[11] Commission on Dietetic Registered dietitian demographics. https://certifications/registered-dietitians-demographics. Updated December 1, 2013. Accessed December 30, 2016.

[12] Mahadev S, Simpson S, Lebwohl B, Lewis S, Tennyson C, Green P. Is dietitian use associated with celiac disease outcomes? Nutrients. 2013;5(5): 1585-1594

[13] Meyer K, Fasshauer M, Nebel I, Paschke R. Comparative analysis of conventional training and a computer-based interactive training program for celiac disease patients. Patient Educ Couns. 2003; 54(3):353-360.

[14] Boyce, B. Nutrition apps: opportunities to guide patients and grow your career. J Academy Nutr Diet. 2014;114(1):13-15.

[15] Celiac Disease Foundation. http://celiac.org. Accessed July 26 2016.

[16] Celiac disease. http://www.digestive.niddk.nih.gov/ddiseases/pubs/celiac. Accessed July 26, 2016.

[17] United States Census Bureau. Table 1159159. Internet activities of adults by community type: 2011

https://www.census.gov/library/publications/2011/compendia/stata b/131ed/information-communications.html. Accessed May 23, 2016.

[18] Norman G, Zabinski M, Adams MA, Rosenberg D, Yaroch A, Atienza A. A review of eHealth interventions for physical activity and dietary behavior change. Am J Prevent Med. 2007;33:336-345.

[19] Nyquist H, Rhee Y, Brunt A, Garden-Robinson J. Promoting health eating and exercise through online messages: a pilot study. J Extension. 2011;49(6):6FEA6.

[20] Aungst TD, Clauson KA, Misra S, Lewis TL, Husain I. How to identify, assess and utilise mobile medical applications in clinical practice. Int J Clin Pract. 2014;68(2):155-162.

[21] Simpson S, Thompson T. Nutrition assessment in celiac disease. Gastrointest Endosc Clin N Am. 2012;22(4):797-809. 